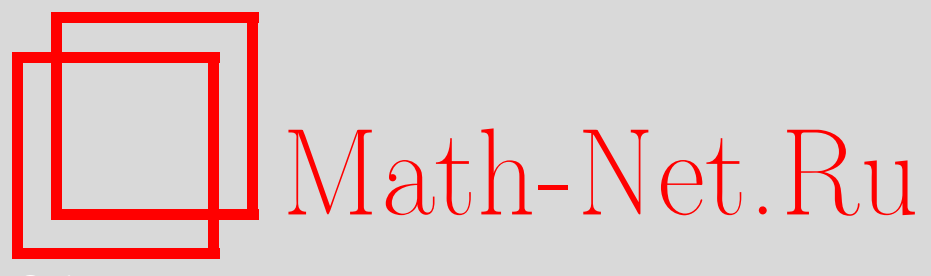

А. Э. Гутерман, Б. Кузьма, Характеризация отображений, строго сохраняющих нули матричных многочленов, УМH, 2008, том 63, выпуск 5, 185-186

DOI: https://doi.org/10.4213/rm9224

Использование Общероссийского математического портала Math-Net.Ru подразумевает, что вы прочитали и согласны с пользовательским соглашением http://www . mathnet.ru/rus/agreement

Параметры загрузки:

IP: 54.198 .64 .247

26 апреля 2023 г., 08:52:32

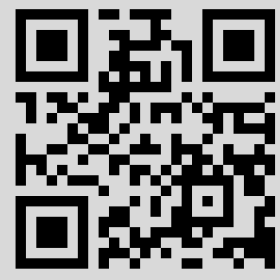




\title{
Характеризация отображений, строго сохраняющих нули матричных многочленов
}

\author{
А. Э. Гутерман, Б. Кузьма
}

Пусть $\mathfrak{p}\left(x_{1}, \ldots, x_{k}\right)$ - произвольный элемент свободной ассоциативной алгебры (многочлен от попарно некоммутирующих переменных $x_{1}, \ldots, x_{k}$ степени $\operatorname{deg} \mathfrak{p}>1$ ) над алгебраически замкнутым полем $\mathbb{F}$ нулевой характеристики, и пусть $\Phi$ : $\mathscr{M}_{n}(\mathbb{F}) \rightarrow \mathscr{M}_{n}(\mathbb{F})$ - линейное биективное отображение пространства матриц размера $n \times n$ с коэффициентами из поля $\mathbb{F}$. Предположим, что для любого набора матриц $\left(A_{1}, \ldots, A_{k}\right)$, удовлетворяющего условию $\mathfrak{p}\left(A_{1}, \ldots, A_{k}\right)=0$, справедливо $\mathfrak{p}\left(\Phi\left(A_{1}\right), \ldots, \Phi\left(A_{k}\right)\right)=0$. Проблема характеризации таких отображений $\Phi$ восходит к работе У. Уоткинса [1], 1976 г.

Эта проблема относится к теории отображений, сохраняющих матричные инварианты и свойства, которая берет свое начало с работ Фробениуса, Шура и Дьёдонне (см. [2]) и является интенсивно развивающимся разделом современной линейной алгебры. Характеризация отображений, сохраняющих нули матричных многочленов, играет центральную роль в этих исследованиях (см. [2; гл. 4]).

В настоящей заметке проблема Уоткинса решена для произвольных однородных полилинейных многочленов, сумма всех коэффициентов которых отлична от нуля. Для решения этой проблемы авторами предложен новый метод, позволяющий решить задачу в более общей постановке, а именно, отказаться как от требования линейности, так и от требования биективности рассматриваемых отображений. Еще одно преимущество этого метода заключается в том, что он работает над полем произвольной характеристики, отличной от двух.

Отметим, что для линейных биективных отображений матриц над алгебраически замкнутым полем нулевой характеристики, сохраняющих множество всех нулей данного многочлена от одной переменной, эту проблему решил Р. Ховард (см. [3]), используя методы и результаты алгебраической геометрии. Как отмечено в работе [4], несмотря на активные исследования в этой области (см. [3]-[11]), последовавшие за работой [3], некоторых продвижений удалось достичь лишь для конкретных многочленов, и вопрос характеризации отображений, сохраняющих нули многочленов нескольких переменных, оставался открытым даже в однородном полилинейном случае.

Зафиксируем произвольный однородный полилинейный многочлен $\mathfrak{p}\left(x_{1}, \ldots, x_{k}\right):=$ $\sum_{\sigma \in \mathscr{S}_{k}} \alpha_{\sigma} x_{\sigma(1)} \cdots x_{\sigma(k)}, \alpha_{\sigma} \in \mathbb{F}$, удовлетворяющий условию $\sum_{\sigma \in \mathscr{S}_{k}} \alpha_{\sigma} \neq 0$.

Будем говорить, что отображение $\Phi: \mathscr{M}_{n}(\mathbb{F}) \rightarrow \mathscr{M}_{n}(\mathbb{F})$ сохранлет нули многочлена $\mathfrak{p}\left(x_{1}, \ldots, x_{k}\right)$, если из $\mathfrak{p}\left(A_{1}, \ldots, A_{k}\right)=0$ следует $\mathfrak{p}\left(\Phi\left(A_{1}\right), \ldots, \Phi\left(A_{k}\right)\right)=0$, и $\Phi$ строго сохраняет нули многочлена $\mathfrak{p}\left(x_{1}, \ldots, x_{k}\right)$, если $\mathfrak{p}\left(A_{1}, \ldots, A_{k}\right)=0$ тогда и только тогда, когда $\mathfrak{p}\left(\Phi\left(A_{1}\right), \ldots, \Phi\left(A_{k}\right)\right)=0$.

Теорема 1. Пусть $\mathbb{F}$ - алгебраически замкнутое поле, $\operatorname{ch} \mathbb{F} \neq 2, n \geqslant 4, k \geqslant 3$. Предположим, что сюрвективное отображение $\Phi: \mathscr{M}_{n}(\mathbb{F}) \rightarrow \mathscr{M}_{n}(\mathbb{F})$ строго сохраняет нули многочлена $\mathfrak{p}\left(x_{1}, \ldots, x_{k}\right)$. Тогда существуют такие изоморфизм поля $\varphi: \mathbb{F} \rightarrow \mathbb{F}$, функиии $\gamma: \mathscr{M}_{n}(\mathbb{F}) \backslash\{0\} \rightarrow \mathbb{F}^{*}:=\mathbb{F} \backslash\{0\}$ и $\mu: \mathscr{M}_{n}(\mathbb{F}) \rightarrow \mathbb{F}$ и обратимая матрица $T$, что

(i) $\Phi(A)=\gamma(A) T A^{\varphi} T^{-1}+\mu(A) E$ для всех $A \in \mathscr{M}_{n}(\mathbb{F})$ или

(ii) $\Phi(A)=\gamma(A) T\left(A^{\varphi}\right)^{\operatorname{tr}} T^{-1}+\mu(A) E$ для всех $A \in \mathscr{M}_{n}(\mathbb{F})$,

здесъ через $X^{\varphi}$ обозначена матрица, элементы которой получаются применением автоморфизма $\varphi к$ элементам матрицы $X$, через $X^{\operatorname{tr}}-$ транспонированная матриц, а, через $Е-$ - диничная матрица.

Работа первого автора поддержана грантом МK-2718.2007.1 Президента РФ, работа второго автора - Министерством науки Словении. 
Заметим, что в случае $k=2$ отображение $\Phi$ может не иметь определенной структуры на достаточно большом множестве матриц, а именно, рассмотрим многочлен $\mathfrak{p}=x_{1} x_{2}$, тогда любое отображение, действующее произвольной перестановкой на множестве обратимых матриц, строго сохраняет нули p. Однако на можестве матриц ранга 1 отображение $\Phi$ имеет вид как в теореме 1 , более того в формулах (i) и (ii) отсутствует второе слагаемое

Для многочленов с нулевой суммой коэффициентов ситуация усложняется тем, что среди таких многочленов содержатся тождества матричной алгебры, сохранение нулей которых не накладывает никаких ограничений на отображение $\Phi$. Вопрос построения базиса тождеств матричной алгебры - открытая проблема (см. [12; проблема 3.2.1]), поэтому не существует конструктивного способа исключить из рассмотрения все тождества. В связи с этим в настоящей работе мы ограничиваемся более узким классом однородных полилинейных многочленов, где суммирование ведется по следующему множеству допустимых перестановок.

ОПределЕниЕ 2 . Пусть $k \geqslant 2$. Множество перестановок $\Xi \subseteq \mathscr{S}_{k}$ называется допустимым, если выполнены следующие условия: (i) существует такое $t \in\{1, \ldots, k\}$, что каждая перестановка $\sigma \in \Xi$ фиксирует первые $t-1$ элементов, но $\sigma(t) \neq t$; (ii) существуют такие числа $w, u, v \in\{1, \ldots, k\}, u<v$, что $\sigma(w)=v$ и $\sigma(w+1)=u$ для всех $\sigma \in \Xi$.

Например, для любой перестановки $\sigma \neq \mathrm{id}$ множество $\Xi:=\{\sigma\}$ является допустимым. Также допустимо множество всех перестановок из $\mathscr{S}_{k}$, переставляющих 1 и 2.

Теорема 3. Пусть $\mathbb{F}$ - произвольное поле, $|\mathbb{F}|>2 u n \geqslant 3, k \geqslant 2$ - целье числа. Пусть $\Xi \subset \mathscr{S}_{k}-\oint$ фикированное допустимое множество перестановок и

$$
\mathfrak{p}\left(x_{1}, \ldots, x_{k}\right):=x_{1} \cdots x_{k}-\sum_{\sigma \in \Xi} \alpha_{\sigma} x_{\sigma(1)} \cdots x_{\sigma(k)}, \quad \alpha_{\sigma} \in \mathbb{F}, \quad \sum_{\sigma \in \Xi} \alpha_{\sigma}=1 .
$$

Тогда произвольное биективное отображение $\Phi: \mathscr{M}_{n}(\mathbb{F}) \rightarrow \mathscr{M}_{n}(\mathbb{F})$, сохраняющее множество нулей $\mathfrak{p}$, сохраняет коммутативность.

Характеризация отображений, сохраняющих коммутативность, получена в рабо$\operatorname{Tax}[9]-[11]$.

\section{Список литературы}

[1] W. Watkins, Linear Algebra Appl., 14:1 (1976), 29-35. [2] Linear Multilinear Algebra, 33:1-2 (1992), 1-129 (Special issues: A survey of linear preserver problems). [3] R. Howard, Linear Algebra Appl., 30 (1980), 167-176. [4] M. A. Chebotar, Y. Fong, P.-H. Lee, Linear Algebra Appl., 48 (2005), 230-243. [5] L. B. Beasley, A. E. Guterman, S.-G. Lee, S.-Z Song, Linear Algebra Appl., 401 (2005), 325-340. [6] K. I. Beidar, Y. Fong, J. Algebra, 217:2 (1999), 650-667. [7] P. Botta, S. Pierce, W. Watkins, Pacific J. Math., 104 (1983), 39-46. [8] M. Brešar, P. Šemrl, Proc. Amer. Math. Soc., 119:1 (1993), 81-86. [9] A. Fošner, Linear Multilinear Algebra, 53:5 (2005), 323-344. [10] T. Petek, Publ. Math. Debrecen, 56:1-2 (2000), 53-61. [11] P. Šemrl, Acta Sci. Math. (Szeged), 71:3-4 (2005), 781-819. [12] V. Drensky, E. Formanek, Polynomial identity rings, Adv. Courses Math. CRM Barcelona, Birkhäuser Verlag, Basel, 2004, 230-243.

\section{А. Э. Гутерман (А. 亡̀. Guterman)}

Московский государственный университет им. М. В. Ломоносова

E-mail: guterman@list.ru

\section{Б. Кузьма (B. Kuzma)}

Institute of Mathematics, Physics and Mechanics, Lyublyana, Slovenia

E-mail: bojan.kuzma@pef.upr.si
Представлено А. В. Михалёвым Принято редколлегией 20.06.2008 